\title{
Open access simulation toolbox for the grid connection of offshore wind farms using multi-terminal HVDC networks
}

\author{
C E Ugalde-Loo*, O D Adeuyi*, S Wang*, J Liang*, N Jenkins*, S Ceballos ${ }^{\star}, M$ Santos*,

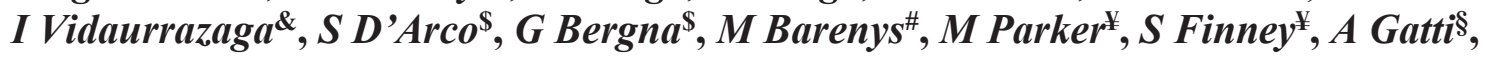

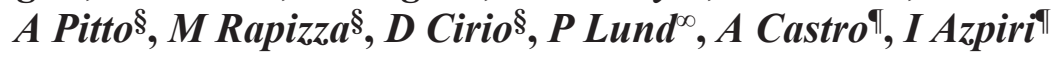

*Cardiff University, Wales, UK (email: Ugalde-LooC@cardiff.ac.uk); \&Tecnalia, Spain; \$SINTEF, Norway; *Gamesa Electric, Spain; ${ }^{\ddagger}$ University of Strathclyde, Scotland, UK; ${ }^{\S} R S E S p A$, Italy; ${ }^{\infty}$ Energinet.dk, Denmark; Iberdrola, Spain

Keywords: BEST PATHS project; Multi-terminal HVDC networks; Wind farms; Open access toolbox; Simulation.

\begin{abstract}
Decarbonisation of the European electricity system can become dauntingly costly due to transmission and distribution network issues arising from the integration of intermittent renewable generation sources. It is expected that wind energy will be the principal renewable source by 2050 and, as such, a number of initiatives in the academia and in the industry are being carried out to propose solutions to best accommodate the wind resource. This paper presents work carried out by DEMO 1 partners within the EU FP7 project BEST PATHS. A MATLAB/Simulink toolbox consisting of the necessary building blocks for the simulation and integration of offshore wind farms using enabling technologies such as multiterminal high-voltage direct-current grids is presented. To illustrate the toolbox capabilities, a number of system topologies is studied. System performance is assessed and measured against a set of key performance indicators. To ensure knowledge dissemination, the toolbox has been made available as open access in the BEST PATHS project website.
\end{abstract}

\section{Introduction}

In Europe, it has been recognised that by 2050 wind energy (particularly the offshore resource) will be the most widely adopted renewable source to contribute towards the abatement of greenhouse gas emissions. A business as usual approach to improve infrastructure will not be sufficient to meet policy objectives at reasonable cost $[1,2]$.

Although several offshore power transmission systems employ high-voltage alternating-current (HVAC), operators and manufacturers are also now considering high-voltage direct-current (HVDC) solutions [3-5]. Such a technology shift can be explained by $i$ ) a higher quality and more reliable wind resource farther away from shore with higher average wind speeds, and thus, $i$ ) longer offshore transmission cables where HVDC becomes more cost-effective than HVAC. It is widely recognised that the integration of large offshore wind farms (OWFs) using HVDC is a feasible option for supplying bulk power over long distances [6].

HVDC links have been deployed to increase cross-country exchange capabilities, with voltage source converter (VSC) based schemes becoming the preferred option [7]. The delivery of smooth and reliable power to onshore AC grids may be facilitated by connecting additional VSCs to form a multi-terminal HVDC (MTDC) grid. It is expected that an MTDC system will not only enable excess energy to be transferred between countries, but will also increase the functionality and reliability of the network [8]. An MTDC grid may reduce the number of converter stations and transmission lines compared to point-to-point links, whilst providing additional flexibility [9-11].

An effective analysis and understanding of future offshore MTDC transmission systems requires accurate modelling and simulation of all components - ranging from wind turbines (WTs) to the DC grid [12]. Special attention should be dedicated to existing $\mathrm{AC}$ onshore grids (particularly to weak and low-inertia systems) and to the different available VSC topologies. Manufacturers normally develop detailed simulations of their equipment separately; however, a standard practice is to use generic models for the components that they do not manufacture; e.g., an HVDC converter vendor may not have access to a detailed WT model or to models from another HVDC vendor. Such an approach may lead to unwanted dynamic interactions between components upon disturbances or changes in operating conditions.

A group of 8 transmission system operators (TSOs), manufacturers, a generator company, and research institutions within the EU FP7 project BEST PATHS are working towards removing technical barriers preventing the largescale penetration of renewable energy production in Europe. To contribute to this effort, in this paper the DEMO 1 partners within the project present a MATLAB/Simulink open access toolbox for the integration of OWFs using MTDC grids. The toolbox has all the necessary building blocks to carry out simulation studies, including VSC stations (both averaged and switched models of modular multi-level converters, MMCs), high-level converter controllers (including AC voltage, DC voltage/reactive power, and active/reactive power control), an AC grid (considering generators, loads, transformers and $\mathrm{HV}$ transmission lines), frequency dependent DC cable models, and a wind farm aggregated model adapted from a real WT (including full power back-to-back converters and internal control algorithms).

To illustrate the capability of the models, different system configurations are simulated. For completeness, system performance is assessed and measured against a set of proposed key performance indicators (KPIs). 


\section{The 'Open Access' Toolbox}

A set of models and control algorithms have been developed. These have been implemented in MATLAB/Simulink and have been published as an open access toolbox in the BEST PATHS website (www.bestpaths-project.eu/). Accompanying documentation has been developed for its correct use.

Although the models and algorithms have been employed to construct, simulate and assess the system topologies presented in Section 3, their portability as basic building blocks will enable researchers and designers to study and simulate any system configuration of choice. A brief description of the toolbox models is provided in this section. A more detailed description can be found in Deliverable D3.1 of the project.

\subsection{Converter Stations}

Averaged and switched models for an MMC have been developed. To accurately represent converter performance, switched modules consider semiconductor commutations which establish the voltage evolution of each submodule capacitor [12]. These models are fast and accurate enough to perform electromagnetic simulations, DC fault studies, total harmonic distortion (THD) and high frequency harmonic analysis, and analysis of the fast interactions and oscillations between the different components of an HVDC grid. The switched model comprises two main blocks:

- Power electronics. It includes submodules with half and full-bridge configurations, arm reactors and an $\mathrm{AC}$ circuit breaker. It is possible to change the number of submodules depending on the desired voltage level.

- Internal controllers:

- Circulating current. It considers four components which determine the circulating current reference. The output circulating voltage is added to the upper and lower phase-arm voltages to calculate the total upper and lower arm voltage references. These are sent to the modulation strategy block $[13,14]$.

- Modulation strategy. References are normalised using a feed-forward approach [15] and sent to a modulator. The upper and lower arm voltage references are normalised with regards to the actual total voltage of the upper and lower arms, respectively.

- Voltage balancing. Specific submodules to be activated to generate reference voltage levels in each arm are selected according to the sense of the current flowing across the arm. An algorithm controlling the number of switching transitions is included.

An equivalent capacitor represents all capacitors of a converter arm in the averaged models. Perfect balancing between the arm capacitors and an equal voltage on each module belonging to the same arm are assumed. Averaged models are thus less accurate than their switched counterparts, but the time required to perform simulations is lower [12].

\subsection{High Level Controller}

A high level controller for HVDC transmission systems has been developed. It allows converter operation in three control modes to cover the main control needs of different topologies:
- AC voltage. In this control mode the converter sets the voltage and frequency [16].

- $\quad$ DC voltage and reactive power $\left(V_{d c}-Q\right)[12,17]$. It employs a cascaded structure. In the inner loop, vector control is used to regulate $\mathrm{AC}$ grid currents using grid voltages as control signals. DC voltage is regulated using the $d$-axis component, with reactive power being regulated with the $q$-axis component. The $d$-axis outer loop is fitted with a DC voltage $v s$ active power droop to enable DC voltage regulation and power flow sharing. A phase-locked loop (PLL) generates reference phase angles for $a b c$-to- $d q$ and $d q$-to- $a b c$ transformations.

- Active and reactive power $(P-Q)[12,17]$. It uses a similar structure as the $V_{d c}-Q$ mode, but active power is regulated using the $d$-axis. An active power $v s$ DC voltage droop has been included to the $d$-axis outer loop for DC voltage regulation and power flow sharing. A PLL is also included to generate reference phase angles.

HVDC systems require a control hierarchy for their correct operation [12], as shown in Figure 1. The dispatch controller selects the control mode of the converter station and manages the operating set points. The high level controller receives reference signals from the dispatch controller depending on the selected control mode. The low level controller receives reference voltages to produce a switching signal for the power electronic devices of the converter.

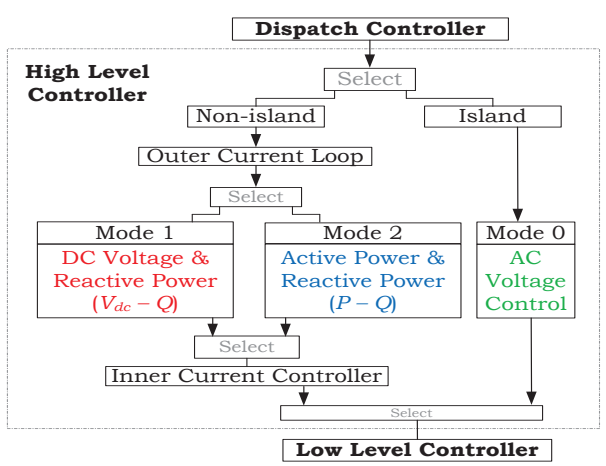

Figure 1. VSC-HVDC control hierarchy.

\subsection{AC Grid}

This is an AC network adapted from the classical 9-bus power system reported in [18] (see Figure 2). It includes:

- Three salient pole synchronous generators, each including a Type 1 exciter, an automatic voltage regulator and a power system stabiliser.

- Three loads modelled as constant $P Q$ absorptions independent from voltage and frequency at the load bus.

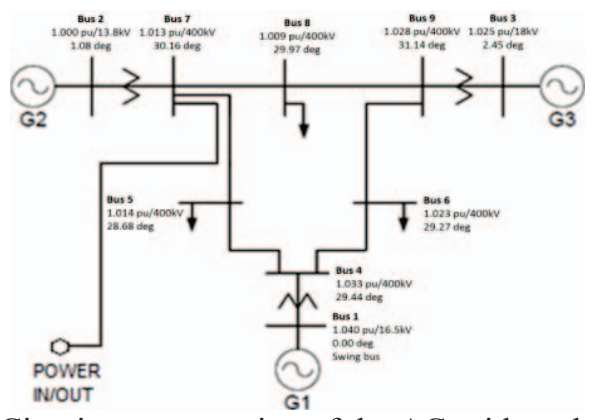

Figure 2. Circuit representation of the AC grid model [18]. 
- Three step-up transformers with configurable winding connections.

- Six high-voltage transmission lines modelled as threephase PI sections with lumped parameters.

A power link has been added to enable the connection of external power circuits such as HVDC networks.

\subsection{Cable}

Simulation of electromagnetic transients on HVDC systems requires frequency-dependent DC cable models. If a small time-step length and a wide-frequency band are required, the travelling wave approach is more efficient than methods using cable models based on PI-sections [19]. However, travelling wave methods are not available in Simulink.

The DC cable section has been modelled as a one-phase, frequency-dependent, travelling wave model. It is based on the universal line model (ULM), which takes into account the frequency dependence of parameters [19]. This has been implemented as a Norton equivalent to model both cable ends via a constant conductance and a controllable current source. Terminal voltages are used as inputs for either a handwritten C-MEX S-function or to an embedded MATLAB function (see Figure 3). The S-function provides the historic current states by implementing ULM subroutines -in turn introduced into the electrical model using the controllable sources.

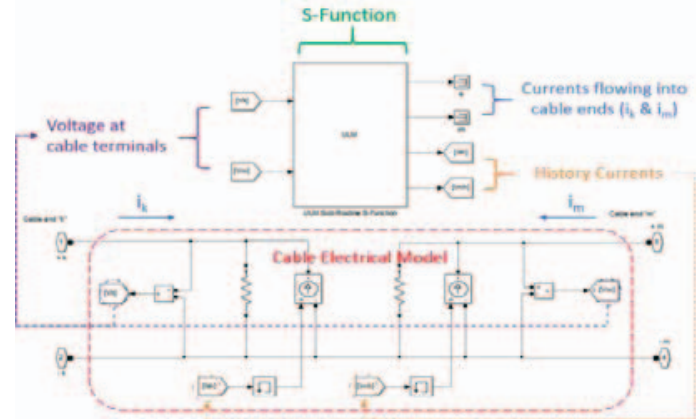

Figure 3. DC cable model using a Norton equivalent.

\subsection{Wind Farm}

The aim of this model is to accurately represent the behaviour of an aggregated OWF. To avoid large simulation times and undesirable computer burden, the following simplifications to the electrical system have been carried out:

- The converter of a wind turbine generator (WTG) is modelled with averaged-model based voltage sources.

- A current source represents the remaining WTGs of the OWF. The current injection of the first WTG is properly scaled to complete the rated power of the whole OWF.

To this end, the first WTG contains:

- A permanent magnet synchronous generator model.

- Averaged models of machine-side converters (MSCs) and grid-side converters (GSCs), including filters and the DC link.

- An LV/MV transformer.

- Internal control algorithms. These include the converter controllers (with the MSC regulating active power and the GSC regulating DC voltage and reactive power), power and rms calculations, grid angle detection using a
PLL, machine angle calculation, dip detection to indicate abnormal operating conditions, and modulation conditioning taking into account the DC voltage level.

The aggregated approach in this model assumes that all WTs within the OWF have the same behaviour. This assumption has been made to reduce the simulation time.

\section{Topologies under Examination}

The open access toolbox presented in this paper aims to contribute to the following objectives within BEST PATHS:

- Improve the knowledge on the integration of OWFs via HVDC links (or future MTDC grids).

- Identify possible interactions between the WTs, converters, HVDC link/grids and the onshore grid.

- Reduce uncertainties from OWFs connected to MTDC and multivendor HVDC schemes and, consequently, derisk the use of these technologies.

To help in meeting these objectives, the HVDC topologies presented in this section have been modelled, simulated and analysed. They constitute likely scenarios to be adopted for the transmission of offshore wind energy in future years.

\subsection{Point-to-Point HVDC Link}

Such a system configuration represents HVDC links under construction nowadays. Power generated by an OWF is transferred to an onshore AC grid, as shown in Figure 4.

\subsection{Three-Terminal MTDC Grid}

This topology, shown in Figure 5, connects three converter terminals to form an MTDC grid. Power is transferred from the two HVDC-connected OWFs to an onshore AC grid.

\subsection{Offshore AC Coupling Configuration}

As shown in Figure 6, the AC outputs of three offshore converter stations are connected to form an offshore AC grid. OWFs are connected to this grid, with offshore converter stations being connected onshore using point-to-point links.

\subsection{Six-Terminal MTDC System with Offshore DC Links}

It includes a six-terminal MTDC grid with two offshore DC links and control systems to transfer power from three OWFs to three onshore AC grids (Figure 7). The converter stations are coupled at the DC side forming an offshore MTDC grid.

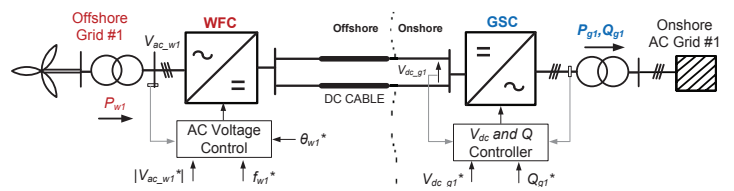

Figure 4. Point-to-point configuration.

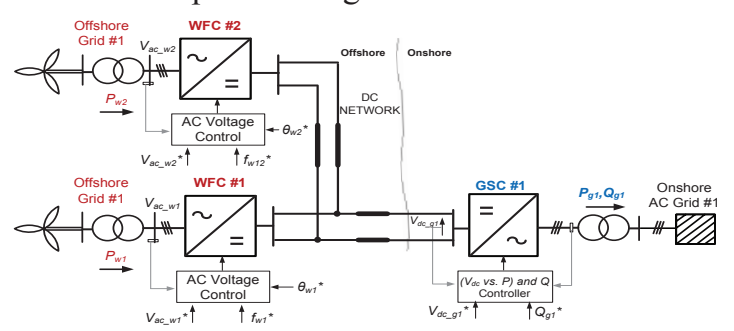

Figure 5. Three-terminal configuration. 


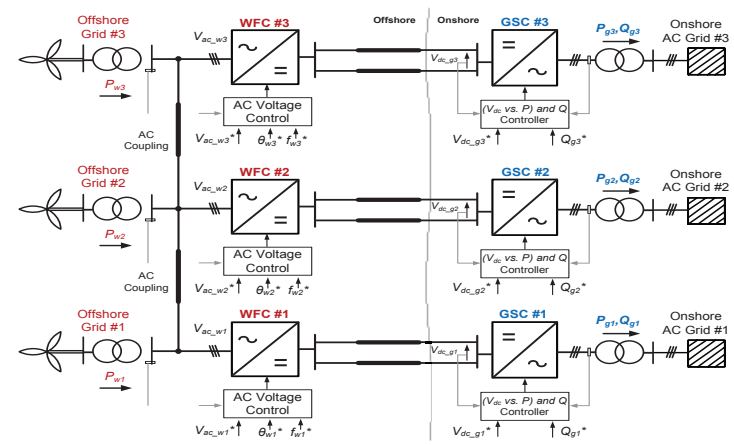

Figure 6. Offshore AC coupling configuration.

\subsection{Offshore Twelve-Terminal MTDC Grid}

In this configuration the DC sides of the converter stations are connected forming a meshed MTDC grid. This topology (not shown due to space limitations) concentrates most of the technical challenges that will be found in the future development of MTDC meshed networks.

\subsection{Interarray}

Power electronics-based topologies for the development of DC inter-arrays have been proposed (not shown due to space limitations). Their study has significant research value as many manufacturers are starting to consider these topologies.

\section{Brief Description of KPIs}

To assess the suitability not only of the toolbox models, but also the performance of prospective future offshore grids, a set of KPIs have been proposed.

\subsection{AC/DC Interactions - Power and Harmonics}

It validates if the proposed converter configurations and controllers achieve an expected performance with respect to:

- Steady state: Measures the steady state error of active power $(<1 \%)$, reactive power $(<1 \%)$, DC voltage at converter terminals $(0 \%$ for constant DC voltage control mode and $<2 \%$ for droop control mode), AC network voltage $(<5 \%)$, offshore AC network frequency $(<1 \%)$.

- $\mathrm{AC}$ and $\mathrm{DC}$ power quality: Establishes if voltages are within limits with respect to $\mathrm{AC}$ voltages harmonics $(<10 \%)$ and DC voltage ripple $(<2 \%)$.

- WT ramp rates: Determines the maximum WTG power ramp rate $(<1 \%)$ to ensure stability after disturbances.

\subsection{AC/DC Interactions - Transients \& Voltage Margins}

This KPI evaluates the transient performance (power flow) of HVDC-connected OWFs during:

- Normal operation. Variations in the wind power and power reallocation between AC nodes. DC link voltage, converter arm current, converter $\mathrm{AC}$ active and reactive power, cell capacitance voltage and converter terminal voltage (at OWFs and AC grid connection) are measured.

- Extreme operation. Considers loss of OWF connection or of DC lines and AC faults at grid terminals. Settling time and variables during normal operation are measured.

The KPI is met if $80 \%$ of all simulation tests are operational and all parameters remain within safe operating limits.

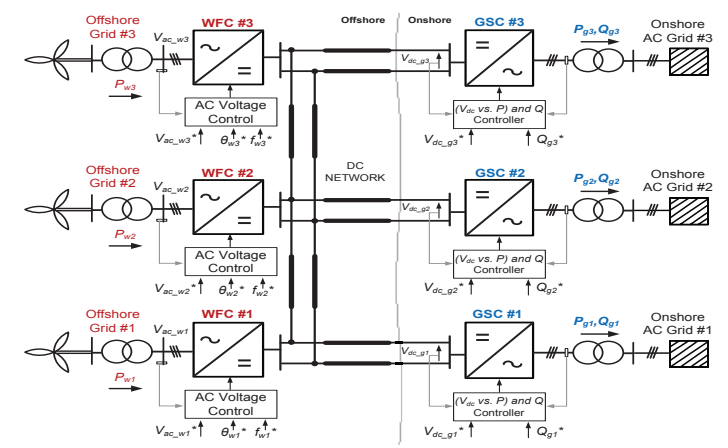

Figure 7. Offshore DC coupling configuration (MTDC grid).

\subsection{Protection Performance / Protection \& Faults}

It validates the functionality and performance of protection systems (for fault location and clearance) upon line-to-ground faults arising at key locations. It is achieved with respect to:

- Protection selectivity: The protection system is able to successfully locate DC faulted lines and clear them.

- Peak current $(<3 \mathrm{pu})$ and clearance time $(<6 \mathrm{~ms})$.

To assess it a hybrid DC circuit breaker model has been built.

\subsection{Interarray Design}

It validates whether DC interarray topologies achieve an adequate performance in terms of OWF security, operation and maintenance. The following aspects are assessed:

- Interarray topology: The maximum number of WTs to conform a DC interarray $(<5 \mathrm{WTs})$.

- Power unbalance $(>3 \%)$.

- Fault tolerance: The number of short-circuits that OWFs should withstand without shutting down $(\geq 1)$.

- Motorising capability: It can deliver and consume active power for maintenance by allowing WT motorising.

\subsection{Resonances}

The KPI is met if $80 \%$ of all simulations are operational and all parameters remain within safe operating limits. An assessment of the following resonances is performed:

- With AC systems: Oscillatory modes between DC converters and $\mathrm{AC}$ grids (including large connected OWFs or synchronous generator-dominated AC grids.

- Internal DC resonance: Oscillatory modes between converter terminals and MTDC networks.

\subsection{Grid Code Compliance}

It evaluates if controllers fulfil the requirements specified in National Grid's Grid Code [20] with regards to:

- Active and reactive power: Establishes if the active and reactive power control of grid-connected converters operate correctly and fulfil frequency and voltage criteria. The steady state error of the active and reactive power $(<5 \%)$ after a defined settling time is measured.

- Fault ride-through: A fault clearance time of $140 \mathrm{~ms}$ during which each converter should remain stable and connected to the system for a three-phase or unbalanced faults in the onshore system. Active power upon fault clearance and within $0.5 \mathrm{~s}$ of voltage restoration at the interface point should be $90 \%$ of the pre-fault value. 


\section{Simulation Results}

To demonstrate the toolbox capabilities, three topologies have been implemented and simulated. Three tests have been carried out, with simulation results presented in Figures 8 to
10. These results correspond, from left to right, to the pointto-point HVDC link (Figure 4), three-terminal MTDC grid (Figure 5), and six-terminal MTDC grid with offshore DC links (Figure 7) topologies. A subset of the KPIs described in Section 4 has been assessed, with a summary given in Table I.
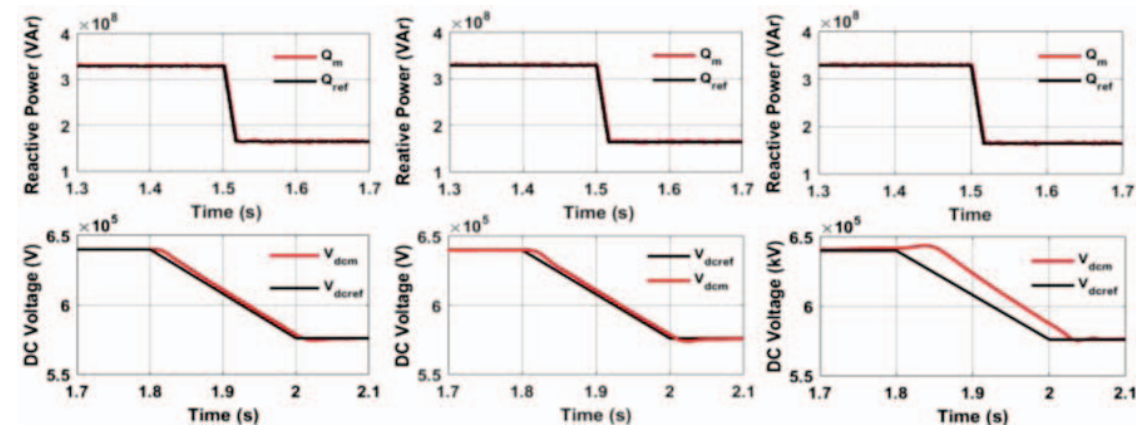

Figure 8. Simulation results for Test 1: $V_{d c}-Q$ control at onshore converter stations.
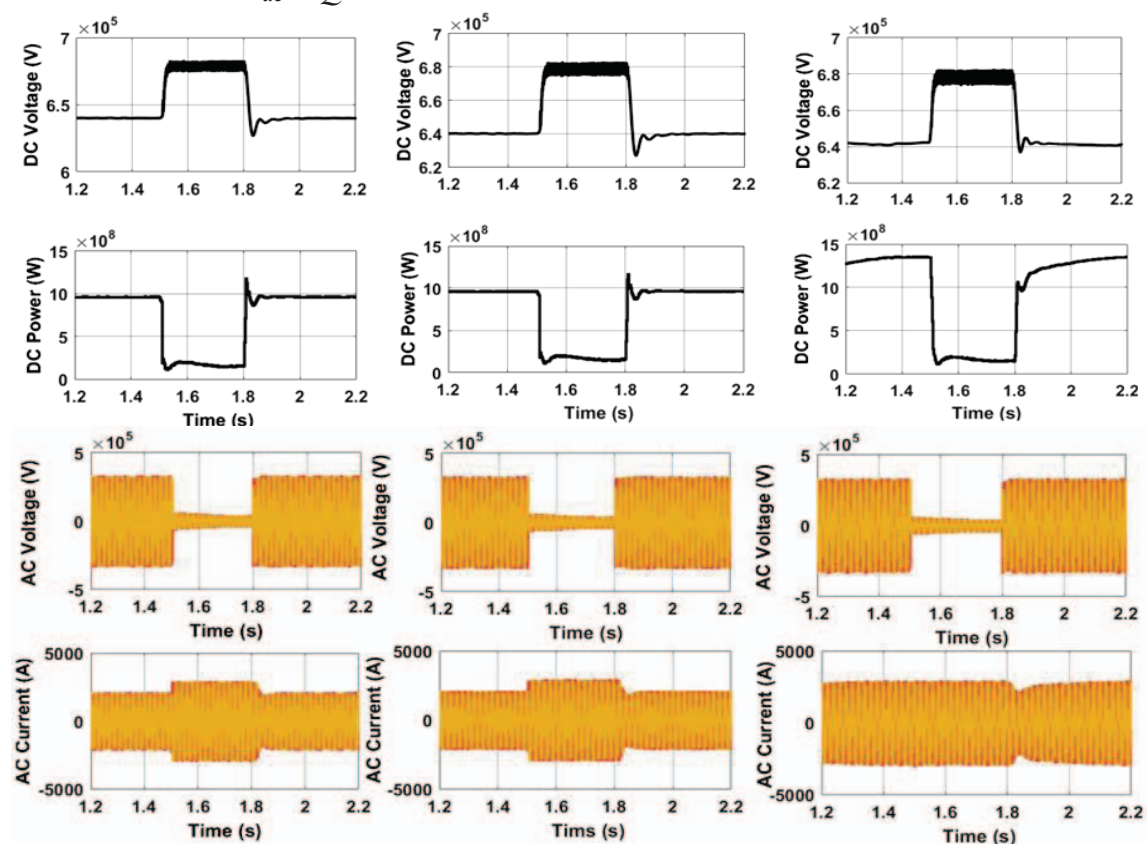

Figure 9. Simulation results for Test 2: Onshore AC fault ride-through capability.
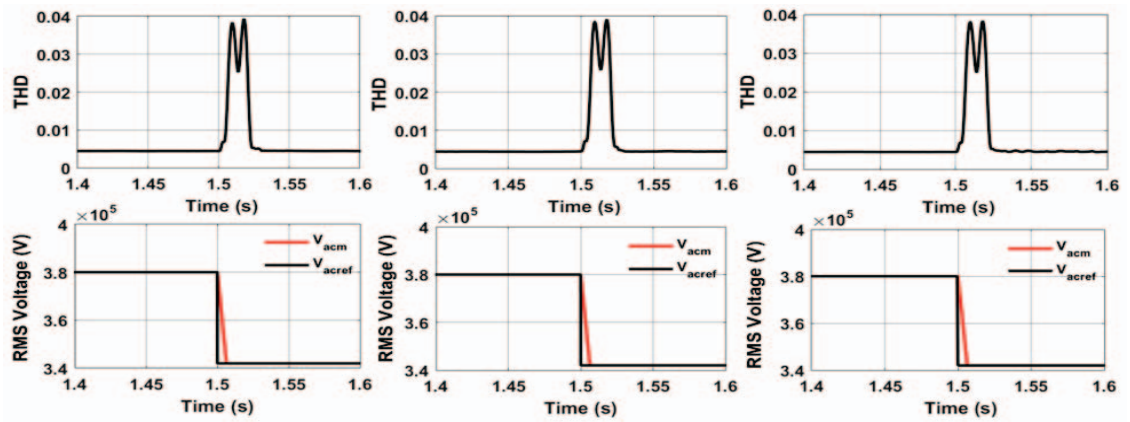

Figure 10. Simulation results for Test 3: Offshore AC voltage THD and converter control performance.

\begin{tabular}{|c|c|c|c|c|c|c|c|c|c|c|c|c|}
\hline \multirow{2}{*}{\multicolumn{2}{|c|}{ KPI tests }} & \multicolumn{2}{|c|}{ Point-to-point link } & \multicolumn{3}{|c|}{ Three-terminal MTDC system } & \multicolumn{6}{|c|}{ Six-terminal MTDC system (offshore DC coupling) } \\
\hline & & WFC & GSC & WFC1 & WFC2 & GSC & WFC1 & WFC2 & WFC3 & GSC1 & GSC2 & GSC3 \\
\hline \multirow{2}{*}{1} & $V_{d c}(\mathrm{sse})$ & - & $0 \%$ & - & - & $0 \%$ & - & - & - & $0 \%$ & $0 \%$ & $0 \%$ \\
\hline & $Q$ (sse) & - & $0 \%$ & - & - & $0 \%$ & - & - & - & $0 \%$ & $0 \%$ & $0 \%$ \\
\hline \multirow{2}{*}{2} & Transient stability & - & stable & - & - & stable & - & - & - & stable & stable & stable \\
\hline & Power Recovery $(90 \%)$ & - & $<0.1 \mathrm{~s}$ & - & - & $<0.1 \mathrm{~s}$ & - & - & - & - & $<0.1 \mathrm{~s}$ & - \\
\hline \multirow{2}{*}{3} & THD (steady state) & 0.0047 & - & 0.0047 & & - & 0.0047 & 0.0047 & 0.0047 & - & - & - \\
\hline & $V_{a c}(\mathrm{sse})$ & $0 \%$ & - & $0 \%$ & & - & $0 \%$ & $0 \%$ & $0 \%$ & - & - & - \\
\hline
\end{tabular}

Table I. KPI assessment for three system configurations. The acronym 'sse' stands for steady-state error. 
In Test 1 , the converter control performance is assessed when references for DC voltage and reactive power are changed. Results are shown in Figure 8. Changes in reactive power $\left(Q_{\text {ref }}\right)$ are requested at $1.5 \mathrm{~s}$ from $1 \mathrm{pu}(330 \mathrm{MVAr})$ to $0.5 \mathrm{pu}$ (165 MVAr) for the onshore GSC in the point-to-point link, GSC in the three-terminal grid and GSC2 in the six-terminal system. DC voltage $\left(V_{d c, \text { ref }}\right)$ is changed at $1.8 \mathrm{~s}$ from $1 \mathrm{pu}(640$ $\mathrm{kV})$ to $0.9 \mathrm{pu}(576 \mathrm{kV})$ for the same converters. As it can be seen, both reactive power and DC voltage match their set points with a negligible steady state error. Thus, the steady state KPI described in Section 4.1 is met.

Test 2 evaluates the onshore AC fault ride-through capability of the HVDC systems. Results are shown in Figure 9. A voltage dip at an onshore grid converter is applied at $1.5 \mathrm{~s}$ during $300 \mathrm{~ms}$ for all topologies, reducing the onshore $\mathrm{AC}$ voltage from 1 to $0.15 \mathrm{pu}$. As it can be seen, this leads to a temporary DC overvoltage, with DC power being reduced significantly. However, all systems remain in operation for more than $140 \mathrm{~ms}$ and DC power recovers within $0.5 \mathrm{~s}$ to $90 \%$ of the nominal value following AC voltage restoration. Thus, the fault ride-through Grid Code compliance KPI presented in Section 4.6 is met.

Test 3 evaluates the total harmonic distortion (THD) of the AC voltage and the converter control performance for offshore AC voltage regulation. Results are shown in Figure 10. The offshore AC voltage (rms) is changed from $1 \mathrm{pu}(380$ $\mathrm{kV})$ to $0.9 \mathrm{pu}(342 \mathrm{kV})$ at $1.5 \mathrm{~s}$. It can be seen that the THD of the controlled offshore AC voltage is maintained below 0.04 during transients and at steady state for all topologies. The measured AC voltage $\left(V_{a c, m}\right)$ follows the set point $\left(V_{a c, \text { ref }}\right)$ without exhibiting steady state error prior and after the reference change. Thus, the steady state and power quality KPIs in Section 4.1 are met.

Due to space limitations it is neither possible to present results for all configurations introduced in Section 3 nor to assess all KPIs in Section 4. However, the results included in this section elucidate the potential and capabilities of the simulation toolbox.

\section{Conclusions}

This paper has presented a MATLAB/Simulink toolbox for the simulation of grid-connected OWFs using HVDC grids. The toolbox models are portable, enabling users to employ them as basic building blocks to assess different topologies. In addition, a set of KPIs to assess system performance and a number of topologies representing future scenarios where OWFs are integrated to onshore grids have been presented. As it has been observed, the simulated configurations exhibit a good performance and the tested KPIs are fully met.

The major contribution of this paper has been providing TSOs, utilities, manufacturers and academic institutions with an open access toolbox to generate the necessary knowledge for the development, construction and connection of MTDC systems -aiming to help de-risking the use of MTDC grids for the connection of OWFs.

\section{Acknowledgements}

The authors gratefully acknowledge the financial support provided by the EU FP7 Programme through the project "BEyond State of the art Technologies for re-Powering AC corridors \& multi-Terminal HVDC Systems" (BEST PATHS), grant agreement number 612748 .

\section{References}

[1] European Commission (EC), “Transmission system operation with large penetration of Wind and other renewable Electricity sources in Networks by means of innovative Tools and Integrated Energy Solutions (TWENTIES)", (2014).

[2] NorthSeaGrid, "NorthSeaGrid: Integrated Offshore Grid Solutions in the North Sea", Policy Brief, (2015).

[3] CIGRE Brochure 533 (WG B4-52), "HVDC Grid Feasibility Study", Paris, (2013).

[4] L. Tang, "Future transmission grids, HVDC and FACTS Systems Aspects", ARPA-E GENI Workshop, (2010).

[5] Siemens, "2 $2^{\text {nd }}$ generation DC grid access for large scale offshore wind farms".

[6] F. Schettler, et al., "Technical guidelines for first HVDC grids", CIGRE Session, Paris, (2012).

[7] National Grid, "Getting more connected - The opportunity from greater electricity interconnection", (2014).

[8] O. D. Adeuyi, et al., "Planning and Operation of the North Sea Grid", HubNet Position Paper Series, (2016).

[9] G. Bathurst and P. Bordignan, "Operating Experience of VSC HVDC - Delivery of the Nan'ao Multi-terminal VSC-HVDC System", CIGRE Symposium, Lund, (2015).

[10] R. Srikandam, P. Kreutzkamp, and J. Decker, "A Selection of Results OffshoreGrid", Seminar on Energy and Climate Policy, Berlin, (2012).

[11] O. D. Adeuyi, et al., "Preventing DC over-voltage in multiterminal HVDC transmission", CSEE Journal of Power and Energy Systems, 1(1), pp. 86-94, (2015).

[12] CIGRE Brochure 604 (WG B4-57), "Guide for the Development of Models for HVDC Converters in a HVDC Grid”, Paris, (2014).

[13] J. Pou, et al., "Circulating Current Injection Methods Based on Instantaneous Information for the Modular Multilevel Converter," IEEE Transactions on Industrial Electronics, 62(2), pp. 777-788, (2015).

[14] D. Soto-Sanchez and T. C. Green, "Control of a modular multilevel converter-based HVDC transmission system," Proc. 14th European Conf. Power Electronics and Applications (EPE), Birmingham, pp. 1-10, (2011).

[15] A. Antonopoulos, L. Ängquist, and H.-P. Nee, "On dynamics and voltage control of the modular multilevel converter," Proc. 13th European Conf. Power Electronics and Applications (EPE), Barcelona, pp. 1-10, (2009).

[16] J. Liang, et al., "Operation and Control of Multiterminal HVDC Transmission for Offshore Wind Farms," IEEE Transactions on Power Delivery, 26(4), pp. 2596-2604, (2011).

[17] O. Anaya-Lara, et al., Wind Energy Generation: Modelling and Control. John Wiley \& Sons, (2011).

[18] P. M. Anderson and A. A. Fouad, Power System Stability and Control. IEEE Press, (1994).

[19] A. Morched, B. Gustavsen and M. Tartibi, "A universal model for accurate calculation of electromagnetic transients on overhead lines and underground cables", IEEE Transactions on Power Delivery, 14(3), pp. 1032-1038, (1999).

[20] National Grid, "The Grid Code", Revision 15, (2016). 